\title{
Applying CIM-to-PIM Model Transformation for Development of Emotional Intelligence Tests Platform
}

\author{
https://doi.org/10.3991/ijoe.v14i08.8747 \\ Karim Arrhioui $\left.{ }^{\bowtie}\right)$, Samir Mbarki \\ Ibn Tofail University, Kenitra, Morocco \\ arr.karim@gmail.com \\ Mohammed Erramdani \\ Mohammed I University, Oujda, Morocco
}

\begin{abstract}
Model driven approach has been introduced to deal with challenges of business and technology. This approach provides tools and elements that permit defining high abstraction level models and metamodels with their transformation to automate code generation. Besides, emotional tests have been introduced to help managing behaviors and relationships between individuals through Emotional Quotient (EQ). In this paper, we propose a model driven approach to generate an emotional intelligence test platform by proposing new CIM metamodel and transformations to generate the PIM as a Class Diagram. We present also a case study that shows how our proposed approach helps generating a class diagram automatically starting from a single input model. This generated model can be easily used to generate the application code.
\end{abstract}

Keywords-Model driven development, Business models, CIM, Model transformations, Emotional Intelligence Test

\section{$1 \quad$ Introduction}

Emotional Intelligence is a new field that has been part of several recent researches in psychology. These researches show that each individual emotionally intelligent has several effective behaviors, aligned actions and balanced perception. However, tests to calculate and measure this emotional intelligence are still in an early phase and do not help deduce concrete results. Indeed, developed tests are at best in their first or second version [1]. That is why developing tests for Emotional Intelligence with its implementation would be very helpful for both employees in their emotional development and for the company for its hiring process which is usually computerized.

In addition to that, Model Driven Engineering has been introduced to overcome the frequent changes of Information Systems evolution. Indeed, MDE approach takes into account the evolution of standards in application areas and provides tools to define high abstraction level models and metamodels with their transformation engines.

In this paper, we present the proposal of combining Model Driven Engineering with Emotional Intelligence and present the set of CIM and PIM metamodels and 
their transformation engine to generate the tests platform. The proposed approach within its metamodels takes into account the major information in a simplified way to help defining the model as instances of CIM. These instances are transformed automatically to PIM class diagrams that can be used to generate the code for any chosen platform.

The paper is organized as follows: In section 2, we outline some works related to Model Driven Engineering and Emotional Intelligence tests. Section 3 presents our MDA approach. We introduce the CIM and PIM metamodels and the transformations engine. A case study, in which we assess the proposed approach, is presented in Section 4 . Finally, section 5 concludes the work and offers further perspectives.

\section{Related work}

In this paper, we combined two fields of research that were the subject of many papers. We find that the paper [2] presents a methodology that is founded on creating a good CIM level, through well-chosen rules, to facilitate transformation to the PIM level. Besides, this models transformation, being essential, was treated using Patterns and Archetypes in [3]. The authors introduced an approach to transform CIM to PIM using UML2 activity diagrams to model the business processes up to the users' tasks. In addition to that, authors of [4] proposed an attempt to define a structure for CIM metamodels that can be divided into a hierarchy of three interconnected models: the business motivation model (BMM), the business process model (BPM), and the requirements model (RM).

On the other hand, several studies dealing with Emotional Intelligence have been introduced. Indeed, some researches presented the development of a measure based on the model of Emotional Intelligence [5] developed by Salovey and Mayer [6]. In [7], the author treated and presented how feelings play a central role in the leadership process and how can the use of emotion help to enhance cognitive processes and decision making. Other authors proposed and tested a theoretical model that integrates several factors such as emotion perception, emotion understanding, and emotion regulation facets [8].

\subsection{Emotional Intelligence Test Platform}

As a major step to start this research, we carried out an analysis of current literature related to both emotional tests field and Model Driven Engineering. It shows that several studies and researches in this direction were conducted.

On one hand, Mayer, Salovey and Caruso have presented the Mayer Salovey Caruso Emotional Intelligence Test (MSCEIT) [9]. This test is constructed through their model based on capacity. For MSCEIT, the normative sample is based on data collected from over 50 research sites with 5,000 participants. The MSCEIT offers two scoring options [1]: 
- The general consensus: where scores are attributed according to the similarity to answers of the normative sample.

- The consensus of experts: scores are attributed according to the similarity to the answers of 21 experts in emotions.

On the other hand, Petrides proposed the emotional intelligence questionnaire (TEIQue) [10]. The normative sample has 1721 individuals. The rating is based on 20 variables (15 facets, 4 factors and the overall feature of EI). Among the facets [1] we find:

- Self-affirmation

- Emotional expression

- Emotional management

- Self-motivation

- The conscience of others

As far as these tests are not automated and taken to an abstracted level, their use is narrowed to specific cases. Besides, not many researches have been proposed to put emphasis on these concepts and give a model that helps generalizing their use to generate a platform.

\subsection{Model Driven Approaches to obtain PIM from CIM}

In the concern of abstraction and automation, Model Driven Engineering was introduced by offering several methods and principles. This approach focuses on developing models of the highest level of abstraction and supports the transformation approach from one model to another.

Several researches have been proposed using this approach to model and generate code and applications in several fields. In [11], the generation of desktop applications is based on one input model. In [12], the proposed approach allows modeling and generating Rich Internet Applications from one input model, and the whole process is automated. Besides, [2] and [3] present new approaches based on defining CIM metamodels and their transformation to PIM metamodels based on patterns and archetypes. These metamodels can be used to propose an abstract metamodel, more generic, to model the emotional tests on one hand, and develop the transformation engine to generate a more technical model in order to have the application fully generated.

From these elements and findings, we propose a combination of the Emotional Tests and MDE approach to present a new metamodel and transformation to generate an application that contains emotional intelligence tests and that can be used by companies and individuals for multiple tasks. 


\section{Model Driven Proposed Approach : From CIM-to-PIM models}

In this paper, we present a model driven approach from CIM to PIM in order to generate an emotional intelligence test platform. Since CIM level of abstraction is the level to start with when working with MDE approach, input elements to define it are related to Emotional Intelligence. For our approach, we designed a new metamodel, as a CIM, that can test the emotional capacity of an individual involved in a business process. This new metamodel takes into consideration all the elements we gathered from our emotional intelligence tests analysis.

Second, we choose an oriented object target platform and we find that Class Diagram is more adapted to go along with the proposed approach. That is why we took a simplified Class Diagram metamodel as PIM second level of abstraction.

Afterward, once metamodels are well defined and validated, the input instances with all emotional activities and elements will be transformed according to the rules defined in the transformation engine into class diagrams respecting the PIM model.

\subsection{CIM Business Model: Emotional Model}

In the proposed CIM metamodel, we integrated the activities that can be present in any process which are related to the person itself acting as a human resource for the whole process. In this way, we can capture the emotional quotient of this resource through a series of questions to ensure the efficient running of the process. The model will make it possible to evaluate the quotient according to the four skills of Goleman [1], to identify the anomalies, then to propose the solutions in the form of choices. The developed metamodel is shown in Fig. 1:

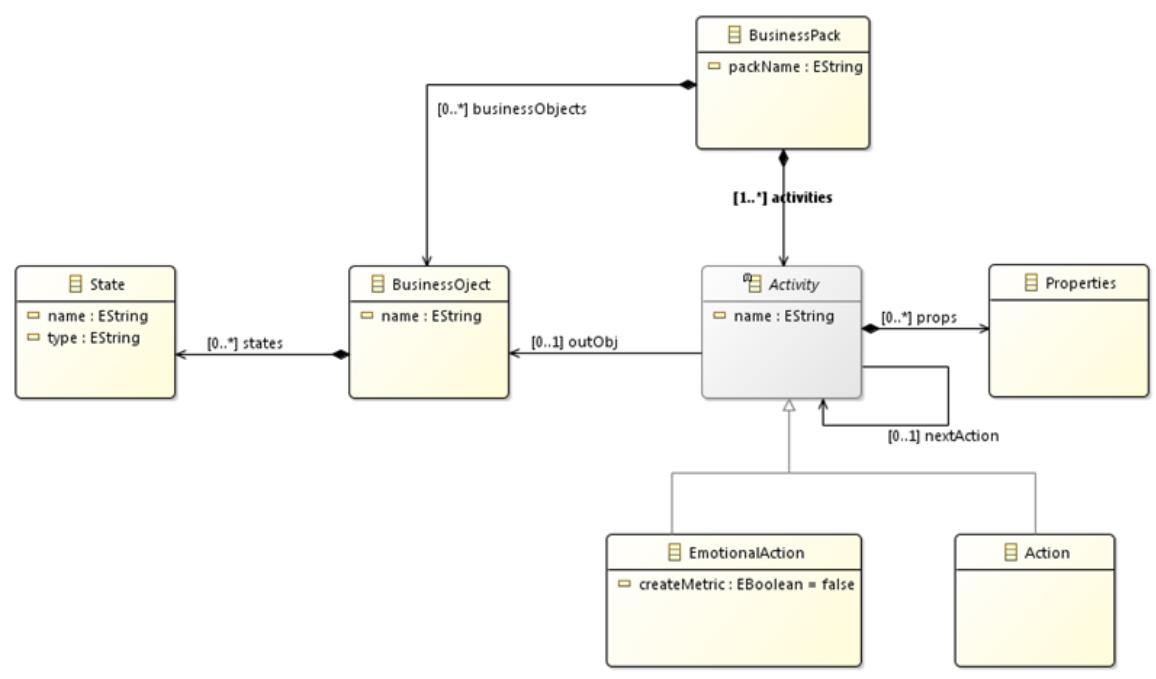

Fig. 1. The CIM Business Model 
Our proposed metamodel gathers the following elements:

- Activity: The main element of the whole metamodel. It captures the activity processed by the resource herself. Each activity is related to a following one, resulting of its execution. We distinguish two categories; Emotional Activity and normal Activity.

- Business Object: It represents an information structure processed and submitted (outObj) by an activity.

- State: It is related to the business object to define its states.

- BusinessPack: The main element that regroups all the sub elements into one common repository.

\subsection{PIM Model: Class Diagram}

Once the input metamodel is defined to get input models properly describing the emotional and normal activities of a resource, the next step is to define the target metamodel as a PIM level. We choose to use a class diagram and refine it, since we aim at generating the code source of the application independently from any execution platform. The simplified class diagram metamodel is shown in Fig. 2:

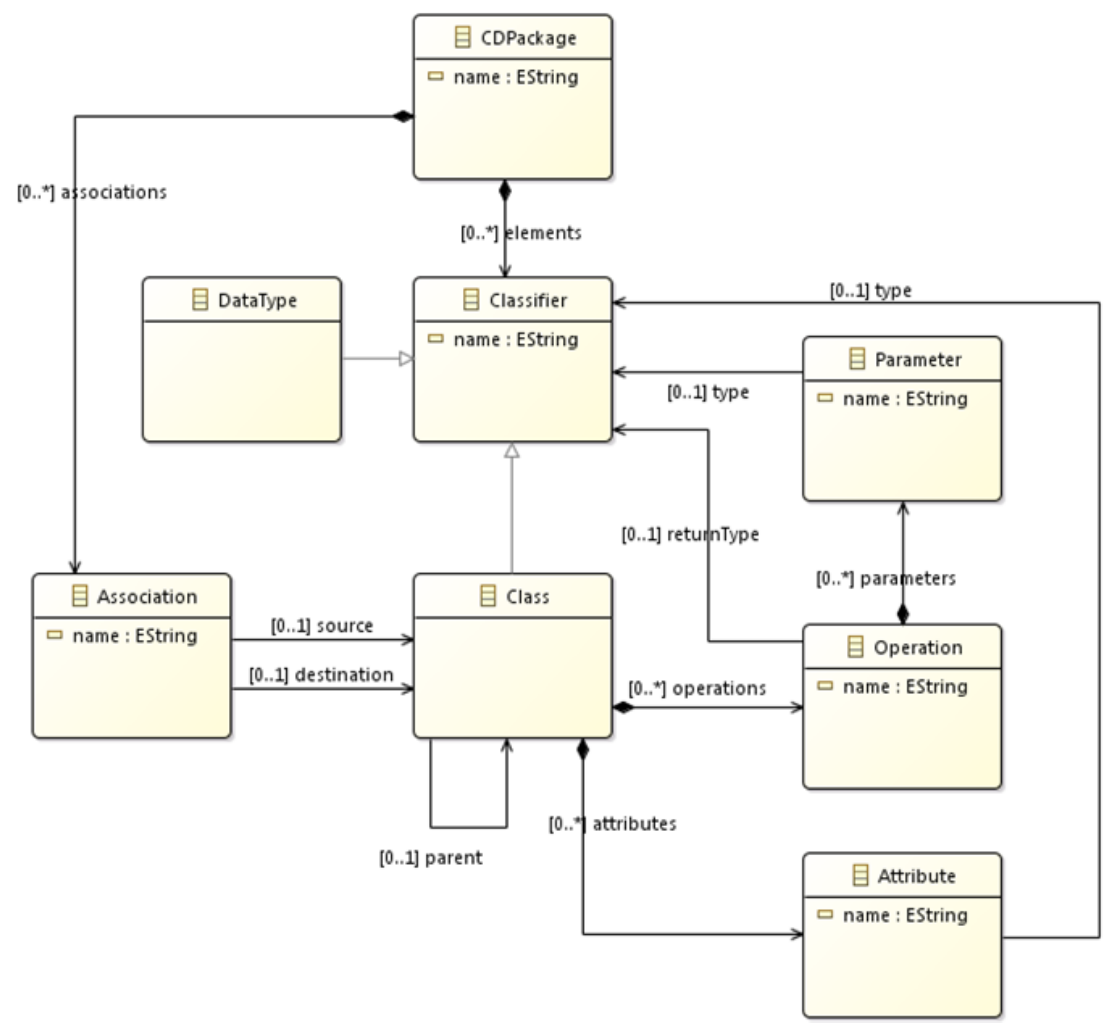

Fig. 2. The simplified class diagram metamodel 
This metamodel contains the following main elements:

- Classifier: meta class that defines generalization hierarchy for Class and Datatype elements.

- Operation: refers to methods that each class offers for business objects

- DataType: is related to the business object to define the state to take.

- CDPack: The main element that regroups all the sub elements into one common repository.

\subsection{Model Transformation}

In order to preserve the modeling approach process of automation, transformation rules should be defined firstly and properly. After that, the implementation is done using the QVT standardized language to get transforming engine. The main transformations are presented in Table 1:

Table 1. The transformation rules

\begin{tabular}{|l|l|l|}
\hline \multicolumn{1}{|c|}{ Source } & \multicolumn{1}{|c|}{ Target } & \multicolumn{1}{c|}{ Transformation } \\
\hline BusinessPack & CDPackage & $\begin{array}{l}\text { BusinessPackToPackage() creates a class diagram package, then, for each } \\
\text { activity, it calls businessObjectToClass(). If the activity is an Emotion- } \\
\text { alAction, it can create a Metric. If nextAction is not null, it calls nextAc- } \\
\text { tionToAssociation() }\end{array}$ \\
\hline BusinessObject & Class & $\begin{array}{l}\text { businessObjectToClass() creates a class with the same name as the busi- } \\
\text { ness object, then calls stateToOperation( })\end{array}$ \\
\hline Activity & Association & $\begin{array}{l}\text { nextActionToAssociation() transforms the nextAction association into an } \\
\text { association between two resulting classes of two business Objects related } \\
\text { to two successive activities }\end{array}$ \\
\hline State & Operation & $\begin{array}{l}\text { stateToOperation() transforms each state of the business object to a } \\
\text { method }\end{array}$ \\
\hline String & DataType & $\begin{array}{l}\text { typeToDataType() creats a data type with the same name as the given } \\
\text { string }\end{array}$ \\
\hline
\end{tabular}

When transforming emotionalAction, if the creatMetric attribute is false, emotionalAction is transformed in the same way as Action, otherwise a new metric is created. It will be attached to the corresponding class of emotionalAction's BusinessObject.

A metric is defined by an ordered series of multiple-choice questions. The user has a fixed number of points per question to evaluate each of the proposed choices. The elements of Metric are shown in the Fig. 3:

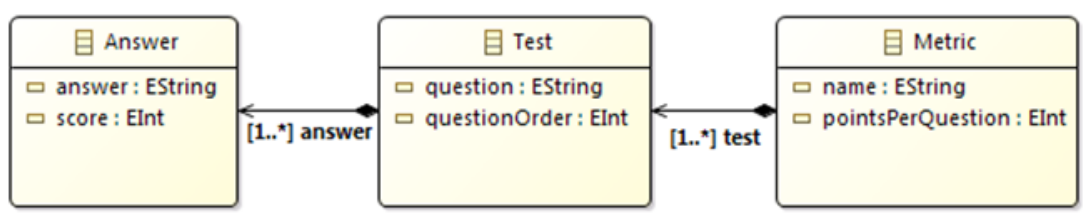

Fig. 3. Metric elements 


\section{Case Study}

In order to validate the proposed approach, we took a case study that handles the monitoring and the evaluation of the emotional quotient of a person involved in a process. For our example, we defined the input model to set an objective, evaluate the candidate emotions through metrics and establish an alternative to be used in case of unpleasant results. For instance, we defined an emotional action "evaluateEmotion" that leads to "establishAlternative" as a result of the action itself. We will be able to generate the metrics related to this transition. The input model is presented in Fig. 4:

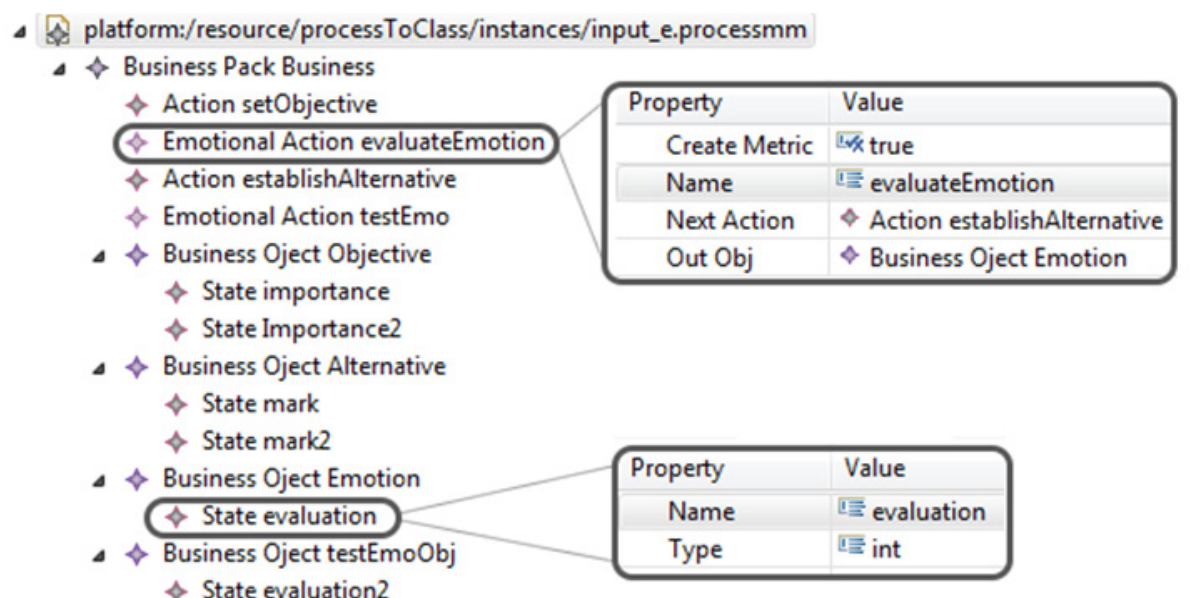

Fig. 4. The input model

This model is then used as an input of the transformation engine and each element gives its related one following transformation rules. The resulting model is a class diagram that contains all the needed classes. It can be used to generate the whole application once the targeted platform is chosen. The output model is shown in Fig. 5.

\section{Conclusion}

In this paper, we based our approach on Model Driven Engineering principles to provide an efficient modeling approach for generation Emotional Intelligence platform. Indeed, we designed a CIM metamodel and defined all the transformation rules to get a PIM level with its class diagram. A case study was presented in order to confirm the effectiveness of the proposed approach.

The future work intended to be improve the CIM metamodel and define the transformation engine from the class diagram to code generation. Besides, we aim at developing a tool to gather the whole elements of the approach and be able to use the approach efficiently. 


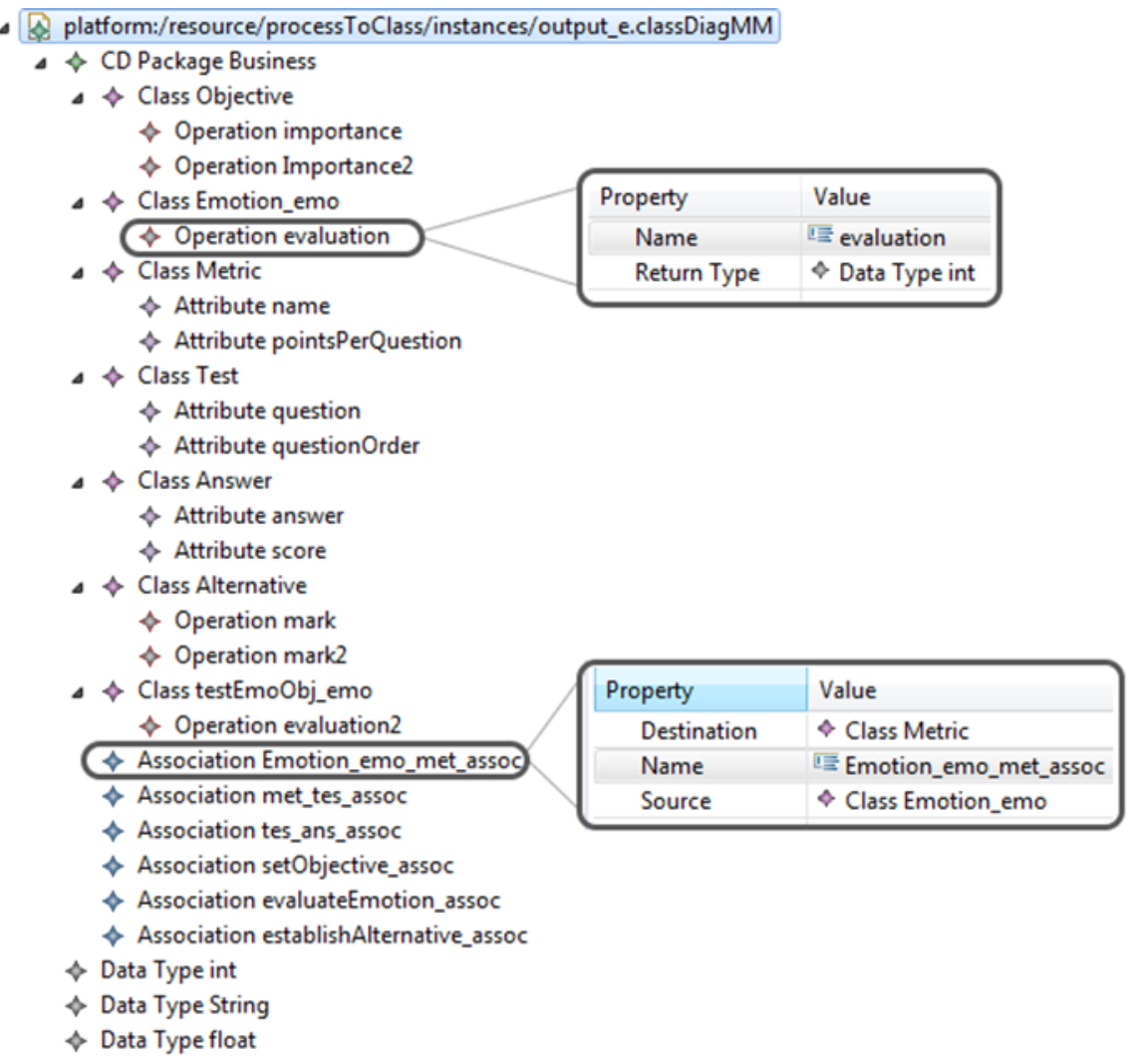

Fig. 5. The generated model

\section{References}

[1] Stough C., Saklofske D. H., Parker J. D. A. (2009). Assessing Emotional Intelligence. Springer.

[2] Rhazali Y., Hadi Y., Mouloudi A. (2015). A Methodology of Model Transformation in MDA: from CIM to PIM. International Review on Computers and Software (IRECOS), 10(12):1186-1201. https://doi.org/10.15866/irecos.v10i12.8088

[3] Kherraf S., Lefebvre É., Suryn W. (2008). Transformation from CIM to PIM using patterns and archetypes. In Software Engineering (ASWEC), 338-346, IEEE.

[4] Kherraf S., Moïse A., Lefebvre É., Suryn W. (2010). Towards a structure for the computation independent model. In Proceedings of the 2nd International Workshop on ModelDriven Architecture and Modeling Theory-Driven Development, 53-59.

[5] Schutte N. S., Malouff J. M., Hall L. E., Haggerty D. J., Cooper J. T., Golden C. J., Dornheim L. (1998). Development and validation of a measure of emotional intelligence. Personality and individual differences, 25(2):167-177.

[6] Salovey P., Mayer J. D. (1990). Emotional intelligence. Imagination, cognition and personality, 9(3):185-211. 
[7] George J. M. (2000). Emotions and leadership: The role of emotional intelligence. Human relations, 53(8):1027-1055. https://doi.org/10.1177/0018726700538001

[8] Joseph D. L., Newman D. A. (2010). Emotional intelligence: an integrative meta-analysis and cascading model. Journal of applied psychology, 95(1):54. https://doi.org/10.1037/ $\underline{\mathrm{a} 0017286}$

[9] Mayer J. D., Salovey P., Caruso D. R., Sitarenios G. (2003). Measuring emotional intelligence with the MSCEIT V2.0. The American Psychological Association, 3:97-105.

[10] Petrides K. V. (2011). Ability and trait emotional intelligence. The Wiley- Blackwell Handbook of Individual Differences, Blackwell Publishing. https://doi.org/10.1002/97814 44343120.ch25

[11] Roubi S., Erramdani M., Mbarki S. (2015). Generating graphical user interfaces based on model driven engineering. International Review on Computers and Software (IRECOS), 10(5):520-528. https://doi.org/10.15866/irecos.v10i5.6303

[12] Roubi S., Erramdani M., Mbarki S. (2016). Modeling and generating graphical user interface for MVC rich internet application using a model driven approach. In Information Technology for Organizations Development (IT4OD), 1-6, IEEE. https://doi.org/10.1109/ IT4OD.2016.7479249

\section{$7 \quad$ Authors}

Karim Arrhioui is a PhD student at MISC (Information Modeling and Communication Systems) laboratory at Ibn Tofail University. He received his computer engineering degree in 2014 from ENSA (National School of Applied Sciences) of Tétouan, Morocco where he studied information systems engineering.

His research topics in the field of model driven architecture focus mainly on metamodeling emotional intelligence tests and metrics.

Samir Mbarki received his B.S. degree in applied mathematics from Mohammed V University, Morocco, 1992, and Doctorate of High Graduate Studies degrees in Computer Sciences from Mohammed V University, Morocco, 1997. In 1995, he joined Ibn Tofail University, Morocco where he is currently a Professor in Department of mathematics and computer science. His research interests include software engineering, model driven architecture and natural language processing.

Mohammed Erramdani teaches the concept of Information System at Mohammed First University. He got his thesis of national doctorate in 2001. His activities of research in the MATSI Laboratory (Applied Mathematics, Signal Processing and Computer Science) focusing on MDA (Model Driven Architecture) and integrating technologies (XML, EJB, MVC, Web Services).

Article submitted 17 April 2018. Resubmitted 18 May 2018. Final acceptance 19 May 2018. Final version published as submitted by the authors. 